\title{
Selection of Optimal Path in Routing Using Genetic Algorithm
}

\author{
Sachin Kumar \\ Department of Computer Science and Applications \\ CH. Devi Lal University, Sirsa, Haryana \\ Avninder Singh \\ Department of Computer Science and Applications \\ CH. Devi Lal University, Sirsa, Haryana
}

\begin{abstract}
Today, the concept of finding the shortest path in network is defined by many researchers using different methods and techniques but to identify the optimal path is a complex problem because in selection of optimal path there may be different logics that can be used to define the optimization of path. In this paper genetic algorithm based approach is proposed and an algorithm has developed to find the optimal path in wired network. Genetic algorithm is considered as search process used in computing to find exact or a near solution for search problems. Population is generated by using permutation encoding scheme and each member of population act as chromosome. Fitness and delay of each chromosome are calculated and crossover and mutation techniques are applied to maintain the diversity of the population. Crossover and mutation collectively offer a search capability that results in better quality of solution.
\end{abstract}

Keywords: Routing, Genetic Algorithm, Optimum Path

\section{INTRODUCTION}

Routing is the process of selecting paths in a network along which to send network traffic. Routing is performed for many kinds of networks, including the telephone network (Circuit switching), electronic data networks (such as the Internet), and transportation networks.

In packet switching networks, routing directs packet forwarding, the transit of logically addressed packets from their source toward their ultimate destination through intermediate nodes, typically hardware devices called routers, bridges, gateways, firewalls, or switches. General-purpose computers can also forward packets and perform routing, though they are not specialized hardware and may suffer from limited performance. The routing process usually directs forwarding on the basis of routing tables which maintain a record of the routes to various network destinations. Thus, constructing routing tables, which are held in the router's memory, is very important for efficient routing. Most routing algorithms use only one network path at a time, but multipath routing techniques enable the use of multiple alternative paths. Routing, in a more narrow sense of the term, is often contrasted with bridging in its assumption that network addresses are structured and that similar addresses imply proximity within the network. Because structured addresses allow a single routing table entry to represent the route to a group of devices, structured addressing (routing, in the narrow sense) outperforms unstructured addressing (bridging) in large networks, and has become the dominant form of addressing on the Internet, though bridging is still widely used within localized environments. (Stallings , 2007)

A routing protocol is a protocol that specifies how routers communicate with each other, disseminating information that enables them to select routes then any two nodes on a computer network, the choice of the route being done by routing algorithms. Each router has a priori knowledge only of networks attached to it directly.

\section{Proposed Algorithm}

\section{Genetic Algorithms: Definitions}

In general, any abstract task to be accomplished can be thought of as solving a problem, which in turn can be perceived as a search through a space of potential solutions. Since we are after "the best" solution, we can view this task as an optimization process. For small spaces, classical exhaustive methods usually suffice: for larger spaces, special A.I. techniques must be employed. GAs are among such techniques. They are cast in different perspectives as follows: 
GA's are stochastic algorithms whose search methods model some natural phenomena: genetic inheritance and Darwinian strive for survival.

- GAs are search algorithms based on the mechanics of natural selection and natural genetics. They combine survival of the fittest among string structures with a structured yet randomized information exchange to form a search algorithm with some of the innovative flair of human search.

- In every generation, a new set of artificial creatures (strings) is created using bits and pieces of the fittest of the old; an occasional new part is tried for good measure. While randomized, GAs are no simple random walk. They efficiently exploit historical information to speculate on new search points with expected improved performance.

- A GA is a method for searching fitness landscapes for highly fit strings.

- The central theme of GAs has been robustness: the balance between efficiency and efficacy necessary for survival in many different environments.

- GAs are a class of general purpose (domain independent) search methods which strike a remarkable balance between exploration and exploitation of the search space.

\section{RESEARCH}

\section{Objective Of Research}

The objective of our study will be to find the optimal path in the network routing. As we have in the biological principle of survival of the fittest, together with the biological evolution, leads to better adaptation of the species to their environment. Here, a local optimum network path is to be found out that suits best in the network. Genetic Algorithm provides us all the feasible solution to reach the destination from the source. There are three basic criteria's in the Genetic Algorithm i.e. selection, cross-over, mutation. Genetic Algorithm provides us best solution to implement the complicated network routing path. This thesis provides us the graphs from the source to destination with the edges as the distance from one node to another with all feasible paths. The goal of optimization is to find the best possible elements $\mathrm{x}$ criteria are expressed as mathematical functions.

The objective of study is

- Study Genetic Algorithm

- Study Matlab language for implementation of Genetic Algorithm in the Network routing

- Study and Analysis the of the optimum routing path in Network

\section{Problem statement}

The problem is to find the optimum path in networks with the help of Genetic Algorithm. From a number of paths between two nodes the optimum path that has least traffic and transfer the packets efficiently is to be find out

\section{Methodology}

We studied and implemented the Genetic Algorithm in Matlab. Real coding with variable length chromosomes has used. The chromosome size is variable for each chromosome and each chromosome represents a probable rout having some distance and total congestion in the path. Number of nodes is fixed in the network with each node having a congestion factor associated to it having value between 0 and $1 ; 0$ represents a totally free node while a 1 represents a totally congested node

\section{Development Environment}

For implementation of Genetic Algorithm we use the Matlab language. The features of Matlab are:

- High-level language for technical computing

- Development environment for managing code, files, and data

- Interactive tools for iterative exploration, design, and problem solving

- Mathematical functions for linear algebra, statistics, Fourier analysis, filtering, optimization, and numerical integration

- 2-D and 3-D graphics functions for visualizing data

- Tools for building custom graphical user interfaces 
- Functions for integrating MATLAB based algorithms with external applications and languages, such as $\mathrm{C}, \mathrm{C}++$, Fortran, Java, COM, and Microsoft Excel. Implementation

Routing is a process of finding paths between nodes. There are mainly two types of routing policies viz., static routing and dynamic routing. In static routing, the routes between the nodes are pre-computed based on certain factors and are stored in routing table. All packets between any two nodes follow the same path. If topology of the network changes, then the path between two nodes may also change, and hence in dynamic routing policy, the routes are not stored but are generated when required. The new routes are generated based on the factors like traffic, link utilization etc which is aimed at having maximum performance. Routing policy may be centralized or distributed. In the case of centralized routing, only centralized node generates routes between any pair of nodes. In distributed routing, each node generates routes independently between pair of nodes as and when required. Other classification of routing policy is optimal routing (global routing) and shortest path routing (local routing). Some of the shortest path algorithms are distance vector algorithm and link state algorithm. Characteristics of routing policy are distributed-ness, stochastic and time varying, multiobjective and multiconstraint.

\section{OPTIMIZATION}

One of the most fundamental principles in our world is the search for an optimal state. It begins in the microcosm where atoms in physics try to form bonds in order to minimize the energy of their electrons. When molecules form solid bodies during the process of freezing, they try to assume energy-optimal crystal structures. These processes, of course, are not driven by any higher intention but purely result from the laws of physics. The same goes for the biological principle of survival of the fittest which, together with the biological evolution, leads to better adaptation of the species to their environment. Here, a local optimum is a well-adapted species that dominates all other animals in its surroundings. Homo sapiens have reached this level, sharing it with ants, bacteria, flies, cockroaches, and all sorts of other creepy creatures. As long as humankind exists, we strive for perfection in many areas. We want to reach a maximum degree of happiness with the least amount of effort. In our economy, profit and sales must be maximized and costs should be as low as possible. Therefore, optimization is one of the oldest of sciences which even extends into daily life. If something is important, general, and abstract enough, there is always a mathematical discipline dealing with it. Global optimization is the branch of applied mathematics and numerical analysis that focuses on, well, optimization. The goal of global optimization is to find the best possible elements $\mathrm{x}$ according to a set of criteria $\mathrm{F}=\{\mathrm{f} 1, \mathrm{f} 2, . ., \mathrm{fn}\}$. These criteria are expressed as mathematical functions, the so-called objective functions.

\section{Classification Of Optimization Algorithms}

In this section, we has discussed a small fraction of the wide variety of global optimization techniques. Before digging any deeper into the matter, I had attempted to provide a classification of these algorithms as overview and discuss some basic use cases. Classification According to Method of Operation is given here.

Figure 3.1 sketches a rough taxonomy of global optimization methods. Generally, optimization algorithms can be divided in two basic classes: deterministic and probabilistic algorithms. Deterministic algorithms are most often used if a clear relation between the characteristics of the possible solutions and their utility for a given problem exists. Then, the search space can efficiently be explored using for example a divide and conquer scheme. If the relation between a solution candidate and its "fitness" are not so obvious or too complicated, or the dimensionality of the search space is very high, it becomes harder to solve a problem deterministically. Trying it would possible result in exhaustive enumeration of the search space, which is not feasible even for relatively small problems. Then, probabilistic algorithms come into play. The initial work in this area which now has become one of most important research fields in optimization was started about 55 years ago.An especially relevant family of probabilistic algorithms are the Monte Carlo based approaches. They trade in guaranteed correctness of the solution for a shorter runtime. This does not mean that the results obtained using them are incorrect - they may just not be the global optima. On the other hand, a solution a little bit inferior to the best possible one is better than one which needs 10100 years to be found. Heuristics used in global optimization are functions that help decide which one of a set of possible solutions is to be examined next. On one hand, deterministic algorithms usually employ heuristics in order to define the processing order of the solution candidates. Probabilistic methods, on the other hand, may only consider those elements of the search space in further computations that have been selected by the heuristic. 


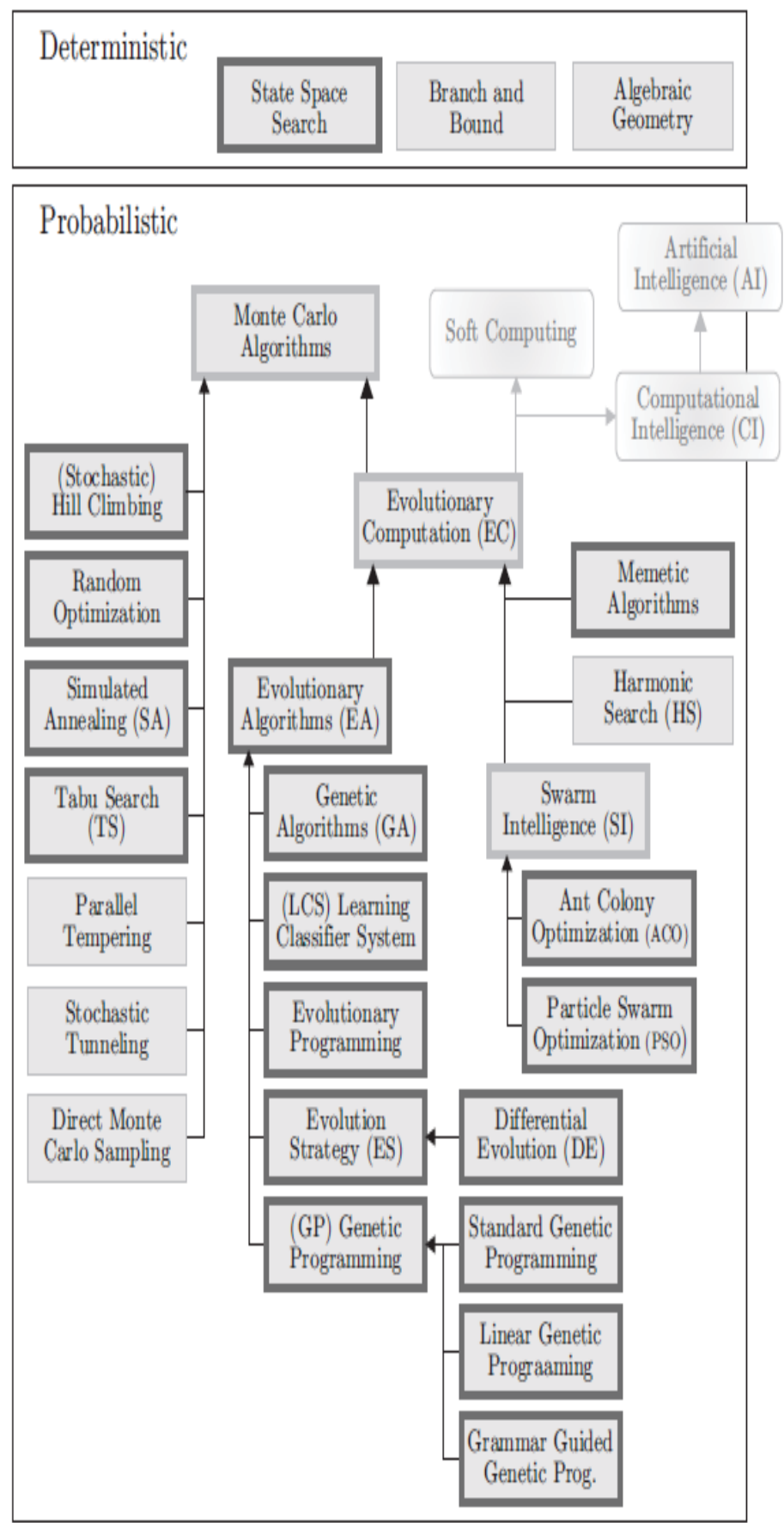

Figure 3.1 The taxonomy of global optimization algorithms.

\section{Implementation Of Proposed Algorithms In Matlab}

The present example is coded in Matlab. Real coding with variable length chromosomes is used. The chromosome size is variable for each chromosome and each chromosome represents a probable rout having some distance and total congestion in the path. Number of nodes is fixed in the network with each node having a congestion factor 
associated to it having value between 0 and 1; 0 represents a totally free node while a 1 represents a totally congested node.. The following process is used to encode the proposed GA for this problem.

\section{CONCLUSION}

The GA is an optimization tool suitable for vast search spaces. We have developed this application of GA using Matlab for static conditions in the Network. That is the congestion factor is taken stationary for the optimization problem at hand, but this application can be programmed for online environment, where congestion factors of the nodes may be changing with time according to the traffic conditions in the network. Other Intelligent tools may be probed in synergism with GA for online applications. GA is specifically suitable for large search spaces, so we can go for optimization of the routing path in networks containing many hundred/thousands or more nodes. For any feasible solution of the problem there is only a binary string corresponding to it. Moreover, a binary string can evidently indicate which nondestination nodes are selected as intermediate nodes.

\section{REFERENCES}

[1] Goldberg D. E., (1989) Genetic Algorithms in Search, Optimization, and Machine Learning. Addison-Wesley, Reading, MA.

[2] Neubauer A., (1997) "The circular schema theorem for genetic algorithms and two-point crossover," in Proc. of Genetic Algorithms in Engineering Systems: Innovations and Applications, pp. 209-214, Sept. 1997.

[3] Holland J.H. (1975) Adaptation in Natural \& Artificial Systems. Ann. Arbor: The Uni. of Michigan press.

[4] Holland J.H. (1962.), "Outline for a logical theory of adaptive systems" J. Assoc. Computer. Mach., vol.3. Pp.297-314.

[5] Michalewicz Z. (1992), Genetic Algorithms + Data Structures = Evolution Programs. Berlin: Springer - Verlag.

[6] Back T.(1992) "The interaction of mutation rate, selection \& self adaptationin genetic algorithm," in parallel problem solving from nature2. Manner R et.al., Eds., Amesterdam, The Neatherland: Elsevier.

[7] Back T. et.al, (1997) "Evolutionary computation: comments on the history \& current state", IEEE Transactions on Evolutionary Computations, vol 1, No. 1.

[8] De Jong K. A. (1975), “An analysis of the behavior of a class of genetic adaptive system”, Ph. D. Dissertation, Univ. of Michigan, Ann Arbor, Diss. Abstr. Int

[9] Davis L, Ed., (1996) Handbook of Genetic Algorithms, New York: Van Norstand Reinhold.

[10] De Jong K. A. (1992), "Are Genetic Algorithms Functions Optimizers?" in Parallel Problem Solving From Nature 2. Amsterdam, the Netherlands: Elsevier. 